\title{
Ann Arbor Stage II Marginal Zone Lymphoma
}

National Cancer Institute

\section{Source}

National Cancer Institute. Ann Arbor Stage /l Marginal Zone Lymphoma. NCI Thesaurus.

Code C68679.

Stage II: Involvement of two or more lymph node regions on the same side of the diaphrag m (II) or localized involvement of a single associated extralymphatic organ or site and its regional lymph nodes with or without other lymph node regions on the same side of the diaphragm (IIE). Note: The number of lymph node regions involved may be indicated by a subscript (e.g., II3). (PDQ) 